\title{
Superdiffusion in Decoupled Continuous Time Random Walks
}

\author{
C. Budde ${ }^{1}$, D. Prato ${ }^{1}$ and M. Ré ${ }^{1}$ \\ ${ }^{1}$ Facultad de Matemática, Astronomía y Fúsica, Universidad Nacional de \\ Córdoba, Ciudad Universitaria, 5010 Córdoba, Argentina \\ prato@mail.famaf.unc.edu.ar \\ (today)
}

\begin{abstract}
Continuous time random walk models with decoupled waiting time density are studied. When the spatial one jump probability density belongs to the Levy distribution type and the total time transition is exponential a generalized superdiffusive regime is established. This is verified by showing that the square width of the probability distribution (appropriately defined)grows as $t^{2 / \gamma}$ with $0<\gamma \leq 2$ when $t \rightarrow \infty$. An important connection of our results and those of Tsallis' nonextensive statistics is shown. The normalized q-expectation value of $x^{2}$ calculated with the corresponding probability distribution behaves exactly as $t^{2 / \gamma}$ in the asymptotic limit.
\end{abstract}


Recently there has been an increasing interest in dynamical processes that display anomalous diffusion 1 - 6 . These phenomena have been characterized by a non linear time dependence of the mean square displacement of the walker

$$
\left\langle x^{2}(t)\right\rangle \sim t^{\alpha}
$$

with $\alpha \neq 1$ ( $\alpha=1$ gives normal diffusion) since $\left\langle x^{2}\right\rangle$ is an usual estimator of the square width of the probability distribution at time $t$. In this way, for anomalous diffusion we have that the probability distribution width grows faster (slower) for $\alpha>1(\alpha<1)$ than it does for normal diffusion. Examples of Eq.(1) with $\alpha<1$ (dispersive diffusion or subdiffusion) are found in disordered media like glasses and fractals structures 70 . Chaotic dynamics and turbulence, on the other hand, give rise to enhanced diffusion (superdiffusion), i.e., $\alpha>1$ 目 4 . Diffusion like behaviour can be modeled in the framework of the continuous time random walk (CTRW), where the central magnitude is the waiting time density (WTD), $\Psi(x, t)$, as was first proposed by Montroll and Weiss 15 . In this way $\Psi(x, t) d x d t$ represents the probability that the walker makes a jump of length $x$ after a time $t$ since the last jump.

When it exists the walker mean square displacement from the origin, at time $t$, can be calculated from the Fourier transform of the spatial probability density $P(x, t)$ according to

$$
<x^{2}(t)>=\int x^{2} P(x, t) d x=-\left.\frac{\partial^{2} P(k, t)}{\partial k^{2}}\right|_{k=0}
$$

where $P(x, t) d x$ is the conditional probability of finding the walker between $x$ and $x+d x$ at time $t$ given that it started at $x=0$ at time $t=0$, and $P(k, t)$ represents its Fourier transform in the space variable. As was shown in previous papers, it is imperative to use a coupled WTD in order to have a finite step mean square displacement in the superdiffusive regime i.e. when the distribution width grows faster than in normal diffusion.

This requirement can be understood since for a decoupled WTD

$$
\Psi(x, t)=p(x) \psi(t)
$$

a divergent mean square displacement for the one step distribution will make infinite $\left\langle x^{2}(t)\right\rangle$ also. This would suggest a formally infinite diffusion coefficient as pointed out by Ott et al.21, 
while the experimental results for elongated micelles analyzed in their paper let them define "a (distance-dependent) diffusion constant. Nevertheless the coupled WTD requirement is not necessary if we consider alternative estimators of the width fo the probability distribution when $\left\langle x^{2}(t)\right\rangle$ diverges, as we will do in this letter.

In particular it is well known that the Levy family of probability densities 11 , has divergent second moment. In spite of this divergence of their second moment a finite width can still be defined for these distributions by considering alternative estimators, such as the inverse of $P(x=0, t)$ or the width at half of the maximun value. Notice that with any of these criteria we get a linear law for the time dependence of the square width in normal diffusion as we do with $\left\langle x^{2}(t)\right\rangle$. One of the most familiar examples of Levy distributions is the Lorentz or Cauchy distribution. This distribution arises naturally in the study of the line shape of fluctuation spectra of stochastic Markov processes2 2 . It is known that the Fourier transform of Levy distributions are, by definition, given by

$$
p(k)=\exp \left(-c|k|^{\gamma}\right), \quad 0<\gamma \leq 2
$$

In the framework of a non-extensive thermostatistics, Tsallis and collaborator䏹 have obtained, from a variational principle, probability densities that share with the Levy family of density functions the asymptotic behavior for large steps. The densities obtained in these papers are (with appropriate simple constraints) the extremal of a functional entropy $23 S_{q}$ where the parameter $q<3$ identifies a particular density. Tsallis' formulation generalizes the classical Boltzmann-Gibbs (obtained for the particular value $q=1$ ) variational formulation. The relationship between parameter $q$ and the exponent $\gamma$ are related by $\gamma=\frac{3-q}{q-1}$ in the range $\frac{5}{3} \leq q<3$, where the second moment diverges, while $\gamma=2$ for $q<\frac{5}{3}$. They also show明 that the $\mathrm{N}$-jump process constructed from these densities scales as $N^{2 / \gamma}$ for $N \rightarrow \infty$.

Our purpose here is to extend the Tsallis' results to a CTRW with a decoupled WTD. One important result of this extension is that in case that the one step probability $p(x)$ (3) is of the Levy type (国), the width of the conditional probability density $P(x, t)$ grows as $t^{1 / \gamma}$ 
for $t \rightarrow \infty$, so that for $\gamma<2$ we obtain a superdiffusive regime.

Let us start by re-obtaining Tsallis' results for the N-jump distribution for a Levy flight. The probability density for the walker position after $N$ steps is given by the N-fold convolution of the one-jump distribution

$$
P(x, N)=p(x) * p(x) * \ldots . * p(x)
$$

whose Fourier transform is

$$
P(k, N)=[p(k)]^{N}=\exp \left(-c N|k|^{\gamma}\right)
$$

by making use of Eq. 4 . Anti-transforming the last equation we finally obtain

$$
\begin{aligned}
P(x, N) & =\frac{1}{2 \pi} \int_{-\infty}^{\infty} d k \exp \left(-c N|k|^{\gamma}\right) \exp (i k x) \\
& =N^{-1 / \gamma} p\left(N^{-1 / \gamma} x\right)
\end{aligned}
$$

where a change of variable has been made.

Eq. (7) is valid for all $\mathrm{N}$ and shows that the width of the N-jump distribution, when appropriately defined, is that of the one-jump distribution times the factor $N^{1 / \gamma}$. The prefactor ensures the normalization condition of the probability density. As it is readily verified, estimators such as the inverse of $P(x=0, t)$ or the width at half of the maximun value, which are finite for these distributions, exhibit the $N^{1 / \gamma}$ growth. In the CTRW scheme a similar result may be obtained when the transition time probability density is

$$
\psi(t)=\lambda \exp (-\lambda t)
$$

In this case

$$
P(x, t)=\frac{1}{2 \pi} \int_{-\infty}^{\infty} d k \exp [-\lambda t(1-p(k))] \exp (i k x)
$$

satisfies the Master equation: 


$$
\frac{\partial P(x, t)}{\partial t}=\lambda \int_{-\infty}^{\infty} d x^{\prime} p\left(x-x^{\prime}\right) P\left(x^{\prime}, t\right)-\lambda P(x, t)
$$

and may be expressed as a power series in time as follows:

$$
P(x, t)=\exp (-\lambda t) \delta(x)+\exp (-\lambda t) \sum_{n=1}^{\infty}\left[\frac{(\lambda t)^{n}}{n !} p\left(n^{-1 / \gamma} x\right)\right]
$$

where $\delta(x)$ is Dirac's delta.

In the limit $\lambda t \gg 1$ only small values of $k$ contribute to the integral in Eq. (9) 16 , giving rise to

$$
P(x, t) \sim \frac{1}{2 \pi} \int_{-\infty}^{\infty} d k \exp \left(-\lambda t c|k|^{\gamma}\right) \exp (i k x) \quad \text { for } \quad \lambda t \gg 1
$$

By making a change of variables we get

$$
P(x, t) \sim(\lambda t)^{-1 / \gamma} p\left[(\lambda t)^{-1 / \gamma} x\right]
$$

then the width of the probability distribution at time $t$ grows as $(\lambda t)^{1 / \gamma}$ in a similar way as was obtained for the discrete time case. As we have mentioned before in normal diffusion $<x^{2}(t)>\sim t$, i.e., the square width grows as $t$, while, in the $\mathrm{N}$-jump Levy process, as we have shown, the square width behaves as $N^{2 / \gamma}$, and as $t^{2 / \gamma}$ in the continuous time case. Since $0<\gamma \leq 2$ we conclude that a superdiffusive regime is established even in the separable CTRW case when the one-jump space distribution belongs to the Levy family.

In Figure 1 we show a plot of the width of the probability distribution versus $\mathrm{t}$ (in logarithmic scale) for various values of the parameter $\gamma$. Here two criteria have been used to define the width of the distributions; one of them just consists in taking the inverse of $P(x=0, t)$, and the other one is the width taken at half of the maximun value of the distribution. We observe that, for $\lambda t \geq 10$, the width grows linearly with $t^{1 / \gamma}$, according to our prediction.

It is worthwhile to notice that our results may be connected with those of Tsallis and collaborators obtained in the framework of nonextensive statistics 1 . we calculate the normalized q-expectation value of $x^{2}$ for a $\mathrm{N}$-jump process 


$$
<x^{2}>_{q}(N)=\frac{\int_{-\infty}^{\infty} d x x^{2}[P(x, N)]^{q}}{\int_{-\infty}^{\infty} d x[P(x, N)]^{q}}
$$

with the following constraint imposed on the single step distribution

$$
\sigma^{2}=\frac{\int_{-\infty}^{\infty} d x x^{2} p(x)^{q}}{\int_{-\infty}^{\infty} d x p(x)^{q}}
$$

Using Eq. (7) and performing a change of variable we obtain

$$
<x^{2}>_{q}(N)=N^{2 / \gamma} \sigma^{2}
$$

This result may be extended to the continuous time case for $\lambda t \gg 1$

$$
<x^{2}>_{q}(t)=(\lambda t)^{2 / \gamma} \sigma^{2}
$$

The last two results suggest that the normalized q-expectation values of $x^{2}$ can be used as an estimator of the square width of the Levy type distributions obtained in 2 , whose normal $(q=1)$ expectation value of $x^{2}$ (second moment) diverges.

To conclude, let us emphasize the main result of this letter: a CTRW, with a separable waiting time density $\Psi(x, t)=p(x) \psi(t)$, whose one-jump space distribution $p(x)$ belongs to a Levy family, gives rise to a generalized superdiffusive process in the case of an exponential form, Eq. (8), for the total transition probability density $\psi(t)$. If, however, $\psi(t)$ has a long time tail the above conclusion may not be true; work along this line is in progress. Finally, the non-extensive thermo statistics seems to be the appropriate framework to tackle anomalous diffusion.

\section{AKNOWLEDGMENTS}

This work was supported by Grants from CONICET-PID 4892 (1998), CONICOR-PID 4624 and 4643 (1998) and SECYT.UNC-PID 163/99 (2000). 


\section{REFERENCES}

${ }^{1}$ C. Tsallis, S.V.F. Levy, A.M.C. Souza and R. Maynard. Phys. Rev. Lett. 75, 3598 (1995). [Erratum: Phys. Rev. Lett. 27, 5442 (1996)].

${ }^{2}$ D. Prato and C. Tsallis. Phys. Rev. E. 60, 2398 (1999).

${ }^{3}$ G. M. Zaslavsky, Physica D, 76, 110 (1994).

${ }^{4}$ J. Klafter, A. Blumen and M.F. Shlesinger. Phys. Rev. A 35, 3081 (1987).

${ }^{5}$ A. Blumen, G. Zumofen and J. Klafter. Phys.Rev. A 40, 3964 (1989).

${ }^{6}$ G. Zumofen, A. Blumen and M.F. Shlesinger. J.Stat.Phys.54, 1519 (1889).

${ }^{7}$ H. Scher and E.W. Montroll. Phys. Rev. B, 12, 2455 (1975).

${ }^{8}$ M.F. Shlesinger. J. Stat. Phys. 10, 421 (1974).

${ }^{9}$ S. Alexander and R. Orbach. J. Phys. (Paris) Lett.43, L625 (1982).

${ }^{10}$ A. Blumen, J. Klafter and G. Zumofen, in Optical Spectroscopy of Glasses, edited by I. Zschokke. (Reidel, Dordrecht. Holland, 1986). p. 199.

${ }^{11}$ A.S. Monin and A.M. Yaglom. Statistical Fluid Mechanics. (MIT, Cambridge, MA, 1971), Vol.I; (1975), Vol. II.

${ }^{12}$ G.K. Batchelor. Proc. R. Soc. London, Ser. A 110, 709 (1926).

${ }^{13}$ S. Grossmann and I. Procaccia. Phys. Rev. A 29, 1358 (1984).

${ }^{14}$ M.F. Shlesinger and J. Klafter. Phys. Rev. Lett. 54, 2551 (1985).

${ }^{15}$ E.W. Montroll and G.H. Weiss. J. Math. Phys. 6,167 (1965).

${ }^{16}$ E.W. Montroll and B.J. West, in Fluctuation Phenomena, E.W. Montroll and J.L. Lebowitz, eds. (North Holland, Amsterdam, 1979).

${ }^{17}$ H. Scher and M. Lax. Phys. Rev. B, 7, 4491; 7, 4502 (1975). 
18 J.K.E. Tunaley. Phys. Rev. Lett. 33, 1037 (1974).

19 J.W. Haus and K.W. Kehr. Phys. Rep. 150, 263 (1987).

${ }^{20}$ G.H. Weiss and R.J. Rubin. Adv. In Chem. Phys. 52, 363 (1983).

${ }^{21}$ A. Ott, J.P. Bouchaud, D. Langevin and W.Urbach; Phys. Rev. Lett. 65, 2201 (1990).

${ }^{22}$ N.G. Van Kampen. Stochastic Processes in Physics ans Chemistry. North-Holland. Amsterdam (1981).

${ }^{23}$ C. Tsallis. J. Stat. Phys. 52, 479 (1988). 


\section{Figure caption}

Figure 1: Width of the probability distribution $P(x, t)$ defined in two ways: $W_{1}=$ width at the half height of $P(x, t)$ and $W_{2}=1 /[P(x=0, t]$ for various one-step Levy distributions

determined by the values of $\gamma$ (see text). Notice the linear behavior of the width, with both criteria, in the asymptotic limit $\lambda t \rightarrow \infty$. The slopes of the curves are consistent with the value $1 / \gamma$ predicted by the theory. 


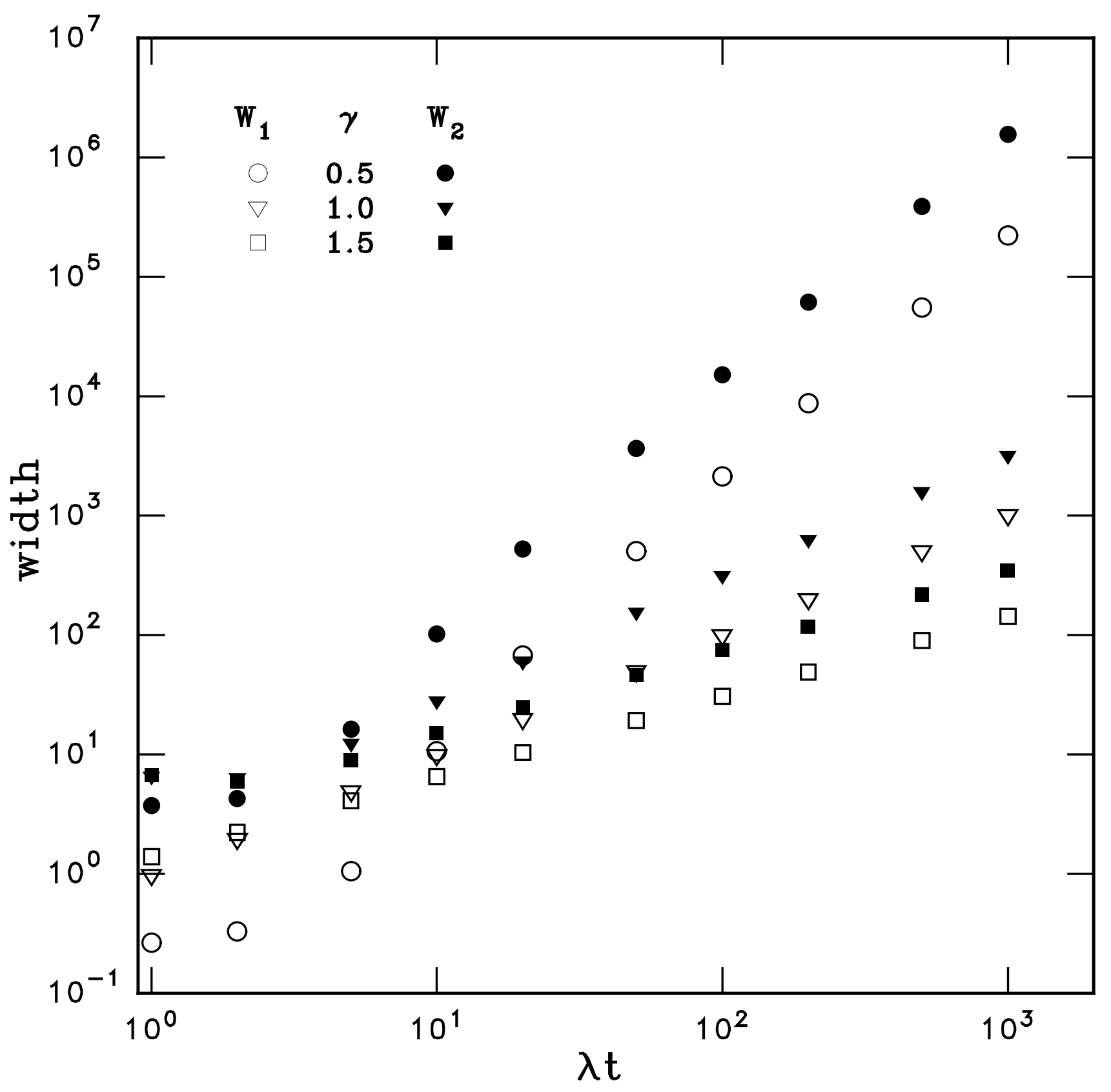

Figure 1 Manuscript Superdiffusion in Decoupled ... Budde, Prato, Re 